\title{
Reverse to proliferation
}

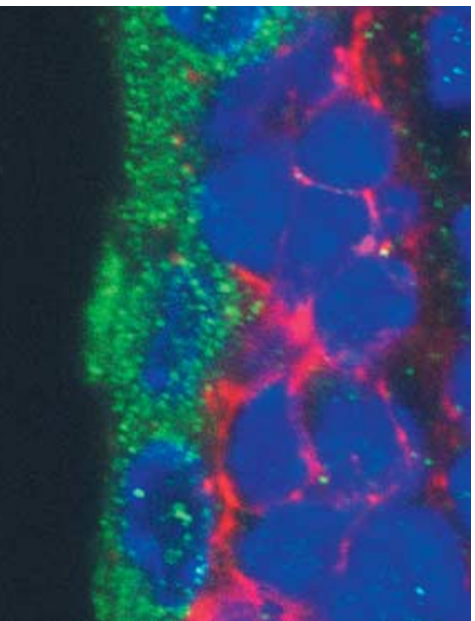

The lateral ventricle wall of the adult mouse brain, labelled with EphA7 (green), ephrin-A2 (red) and a nuclear stain (blue). Image courtesy of J. Frisén, Karolinska Institute, Sweden.
The number of cells in an organ is mainly controlled at the level of proliferation and apoptosis by mitogens and trophic factors. Now, Holmberg and colleagues have identified a novel mechanism whereby the number of neurons in the adult brain is negatively regulated by EphA7-induced ephrin-A2 reverse signalling in neural progenitors.

Ephrins and their Eph tyrosine kinase receptors are important regulators of developmental processes such as axon guidance, cell migration and synapse formation, but their roles in the adult organism are not clear. In the adult mouse brain, ephrin-A2 and EphA7 are expressed in a complementary and mutually exclusive pattern in the lateral wall of the lateral ventricles - one of the neural stem cell niches in the adult brain that can give rise to neurons.

Perturbation of ephrin-A2EphA7 signalling by infusion of the soluble form of either protein increases the number of proliferating cells in the lateral ventricular wall. In mice that lack ephrin-A2 or EphA7, cells in the neural stem cell niche proliferate more and have a shorter cell cycle, which indicates that ephrin-A2 and EphA7 are negative regulators of proliferation.

Ephrins and Ephs can mediate bi-directional signalling - either through Eph receptors (forward signalling) or through membranebound ephrin ligands (reverse signalling). But which signalling pathway is important for neurogenesis? In ephrin-2A-knockout mice, EphA7-expressing cells in the lateral ventricular wall remain quiescent; whereas cells that express progenitor markers - which would normally be co-expressed with ephrin-A2 in wild-type animals show increased proliferative activity. In addition, overexpression of a truncated EphA7 receptor in wildtype neurospheres leads to a significant decrease in proliferation an effect that is not seen in the absence of ephrin-A2. Holmberg et al. conclude that the negative regulation of proliferation by EphA7 and ephrin-A2 is mediated by reverse signalling.

The majority of the neurons that are born in the lateral ventricle wall migrate to the olfactory bulb. In ephrin-A2-knockout mice, there is an increased number of newborn cells in the adult olfactory bulb, which indicates that the proliferative

\section{Seasons of desire}

Melatonin is a key hormone that regulates the sleep-wake cycle. Its expression cycles through the day, with changes in the availability of light, and through the year, with changes in the length of the day. It is thought that melatonin is important in the consolidation of sleep, but its action in the brain is not clear. Now, a new study reports the surprising finding that melatonin directly induces the expression of gonadotropin-inhibitory hormones $(\mathrm{GnIH})$ in the avian brain.

$\mathrm{GnIH}$, which is present in both birds and mammals, including humans, is the only neuropeptide identified so far that antagonizes the effect of gonadotropinreleasing hormone on the production of sex steroids such as oestrogen, progesterone and testosterone. According to the current dogma, seasonal changes in melatonin are not involved in the timing of reproduction in birds. However, Ubuka and colleagues thought this might not be the whole story, as several studies have shown that melatonin can reduce gonadal activity and gonadotropin secretion.
To investigate whether melatonin has an effect on reproduction, they surgically removed the main sources of melatonin the pineal gland and eyes - from the Japanese quail, and measured GnIH expression in the diencephalon. In the absence of melatonin, the levels of $\mathrm{GnIH}$ mRNA and peptide drop markedly in neurons in the paraventricular nucleus $(\mathrm{PVN})$ and their terminals in the median eminence. This effect can be reversed by restoring melatonin in these birds, which indicates that the hormone is involved in regulating the synthesis of GnIH.

Melatonin is only expressed at night, and the level of expression depends on the length of the dark phase of the light-dark cycle. To analyse the effect of endogenous melatonin on reproduction, the authors raised male quails under different lighting conditions. Quails that are raised with simulated short days show higher levels of melatonin and GnIH expression than those that are raised with longer periods of light. In addition, the testicular weight of birds raised in short-day conditions is significantly decreased.
So, how does melatonin regulate $\mathrm{GnIH}$ synthesis in the PVN? Ubuka et al. found that a melatonin receptor subtype, $\mathrm{Mel}_{1 \mathrm{C}}$, is expressed in $\mathrm{GnIH}$-expressing neurons in the PVN, and that melatonin binding is significantly higher in the PVN than in surrounding areas. This indicates that melatonin might directly stimulate the production of GnIH in PVN neurons by binding to their receptors.

Interestingly, in Siberian hamsters the expression of the mammalian homologue of $\mathrm{GnIH}$ in the brain is also regulated by melatonin. Therefore, GnIH might be an important mediator that translates photoperiodic information, in terms of the melatonin expression level, into an effect on reproduction in both birds and mammals.

\section{Jane Qiu}


activity early in the lineage is an important determinant of the number of neurons that integrate the target tissue. Disruption of the EphA7-ephrin-A2 reverse signalling by infusion of soluble ephrin-A2 disinhibits proliferation and results in increased neurogenesis in the adult brain.

It will be important to determine whether there is a similar mechanism in other stem cell populations in which ephrins and Ephs are also expressed. Manipulating the ephrin-Eph signalling could be a potential therapeutic strategy in regenerative medicine and cancer treatment.

Jane Qiu

\begin{abstract}
(0) References and links
ORIGINAL RESEARCH PAPER Holmberg, J. etal. Ephrin-A2 reverse signaling negatively regulates neural progenitor proliferation and neurogenesis. Genes Dev. 19, 462-471 (2005)

WEB SITE

Frisén's laboratory:

http://www.cmb.ki.se/projektdokument/frisen3.htm
\end{abstract}

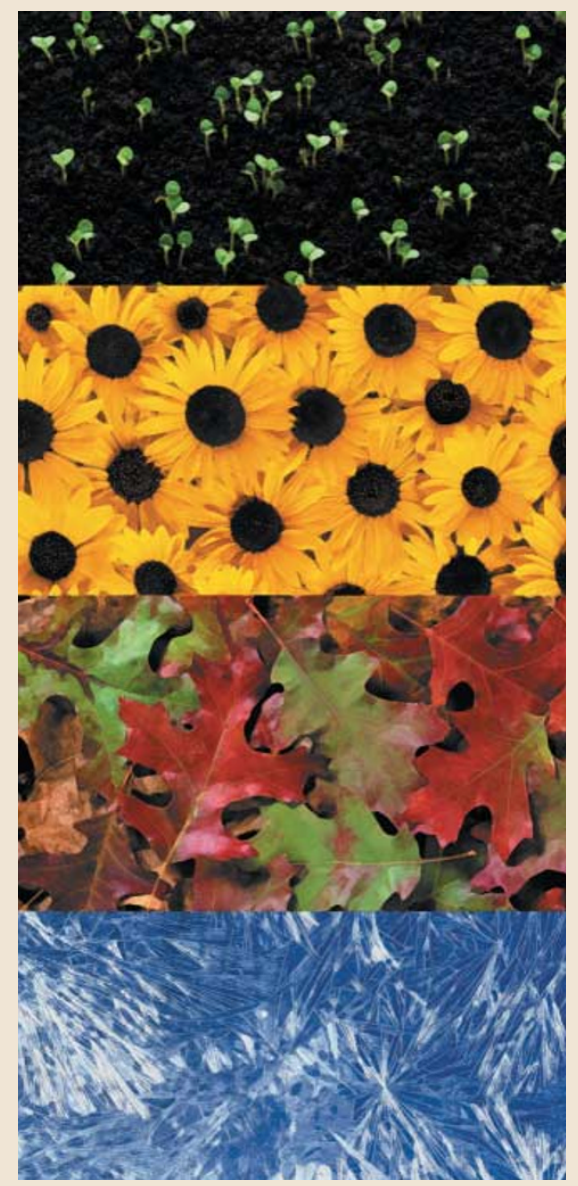

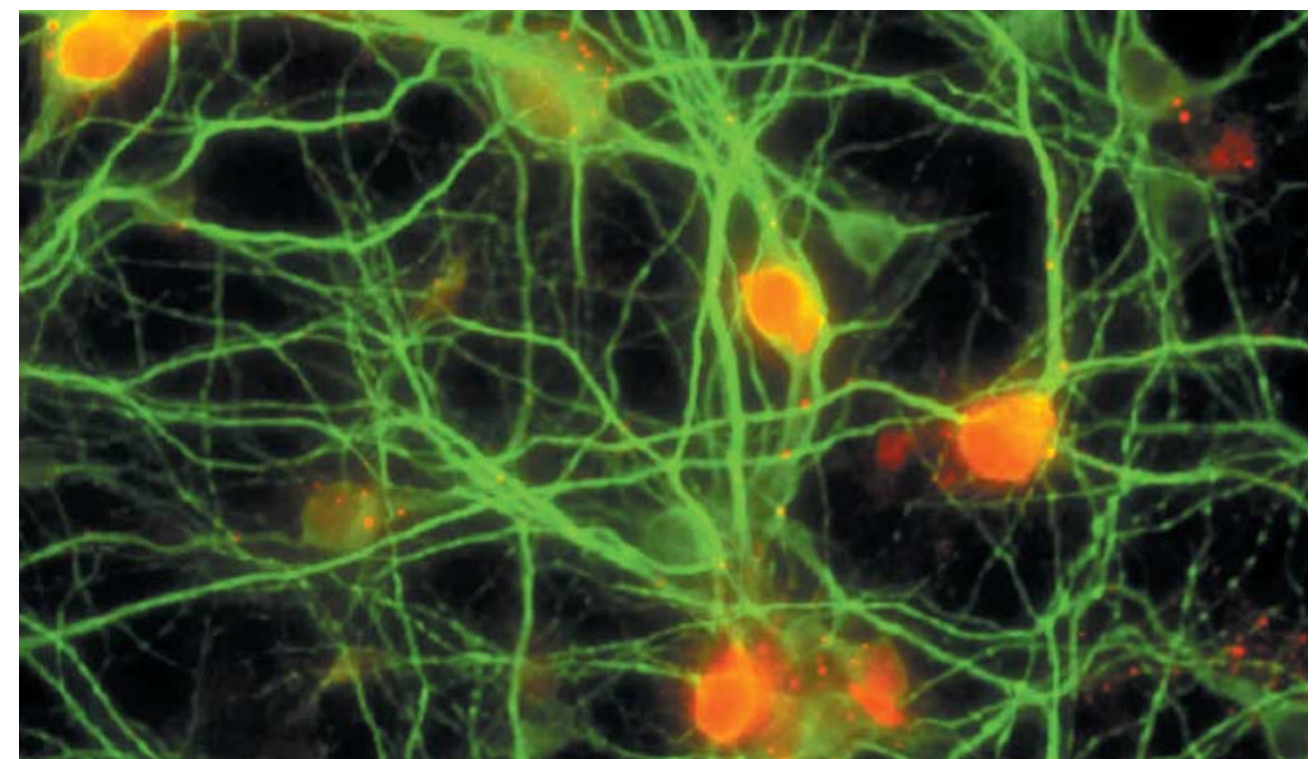

Immunofluorescence analysis of lentiviral vector-mediated $\beta$-galactosidase expression (red) and microtubule-associated protein 2 (green) in primary cortical neurons derived from mice expressing mutant human SOD1. Reproduced, with permission, from Ralph, G. S. et al. Nature Med. 13 March 2005 (doi:10.1038/nm1205).

\section{NEURODEGENERATIVE DISEASE}

\section{ALS meets RNAi}

The idea of using RNA interference (RNAi) as a therapeutic strategy is gaining momentum. Two papers in the April issue of Nature Medicine show that RNAi interferes with the progression of pathology in an animal model of amyotrophic lateral sclerosis (ALS) - a fatal

neurodegenerative disease that attacks both upper and lower motor neurons.

RNAi is a post-transcriptional mechanism of gene silencing that is mediated by small interfering RNA molecules (siRNAs) - 19- to 23nucleotide double-stranded RNA duplexes that promote the cleavage of specific mRNAs. Longlasting RNAi-mediated gene knockdown can be achieved using lentiviral vectors that express the siRNAs. In the two studies, by Raoul et al. and Ralph et al., the authors independently tested whether RNAi could be used to silence mutant forms of the human superoxide dismutase 1 gene (SOD1) expressed in mice. Familial forms of ALS are associated with mutations in SOD1, and mice that overexpress mutant $S O D 1$ show features of the human disease, such as motor neuron death and motor dysfunction.

Both studies showed that the injection of the lentiviral vectors led to substantial delays in the onset and progression of the disease. RNAi reduced the expression of mutant $S O D 1$ in culture and in vivo, prevented the death of spinal and brainstem motor neurons and improved the motor performance of the mice in different behavioural tasks. Raoul et al. also measured electromyographic responses in their animals, and found them to be preserved in the mice treated with the lentiviral vectors.

Early studies on the therapeutic use of siRNAs used vector-delivery methods that have limited potential as treatment agents: hydrodynamic delivery, intracerebral injection and other techniques. More recent papers have added credibility to the idea of using RNAi as therapy by placing extra emphasis on the way in which the vectors are administered. In this regard, the paper by Ralph et al. is particularly compelling, as the authors delivered the lentiviral vector through intramuscular injection, an administration method that has clear therapeutic relevance.

The therapeutic benefit reported in these two papers is among the most substantial ever seen in this animal model, raising hopes for its successful translation to the clinical domain. However, familial forms of ALS account for a very small fraction of clinical cases. So, the need for therapeutic approaches for both familial and sporadic forms of ALS is as pressing as ever. Juan Carlos Lopez, Chief Editor, Nature Medicine

\section{(4) References and links}

ORIGINAL RESEARCH PAPERS Raoul, C. et al. Lentiviral-mediated silencing of SOD1 through RNA interference retards disease onset and progression in a mouse model of ALS. Nature Med. 13 March 2005 (doi:10.1038/nm1207) | Ralph, G. S. et al. Silencing mutant SOD1 using RNAi protects against neurodegeneration and extends survival in an ALS model. Nature Med. 13 March 2005 (doi:10.1038/nm1205) FURTHER READING Cleveland, D. W. \& Rothstein, J. D. From Charcot to Lou Gehrig: deciphering selective motor neuron death in ALS. Nature Rev. Neurosci. 2, 806-819 (2001) | Bossy-Wetzel, E. et al. Molecular pathways to neurodegeneration. Nature Med. 10 S2-S9 (2004) 